\title{
Mitochondrial toxicity of aluminium nanoparticles in comparison to its ionic form on isolated rat brain mitochondria
}

\author{
Arab-Nozari $\mathrm{M}^{1,2}$, Zamani $\mathrm{E}^{3}$, Latifi $\mathrm{A}^{1,2}$, Shaki $\mathrm{F}^{1,2}$ \\ Pharmaceutical Science Research Center, Hemoglobinopathy Institute, Mazandaran University of Medical \\ Sciences, Sari, Iran. fshaki.tox@gmail.com
}

\begin{abstract}
OBJECTIVES: The aim of this study was to evaluate the toxic effect of AINPs on rat brain mitochondria and compare it with that of aluminium's ionic form.

METHODS: Mitochondria were isolated from rat brain. Isolated mitochondria were treated with normal saline (Control) and different concentrations of aluminium ions (Alls) and AINPs (50, 100 and $200 \mu \mathrm{M})$. Then, the effect of AINPs on electron transport chain complexes as well as various endpoints such as mitochondrial oxidative damage (reactive oxygen species, lipid peroxidation, glutathione, and protein carbonyl) and mitochondrial function were assessed. Also, apoptosis was evaluated by cytochrome c release, mitochondrial membrane potential and swelling.

RESULTS: When compared to the control group, the exposure to AINPs showed a marked elevation in oxidative stress markers and inhibition of complex III which was accompanied by disturbance in mitochondrial function. Also, AINPs induced a significant collapse of mitochondrial membrane potential, mitochondrial swelling, and cytochrome $c$ release.

CONCLUSIONS: The comparison of mitochondrial toxicity markers between both forms of aluminium revealed that the toxic effect of AINPs on isolated brain mitochondria was substantially greater than that that caused by Alls, which can probably be ascribed to its higher reactivity (Tab. 1, Fig. 8, Ref. 45). Text in PDF www.elis.sk. KEY WORDS: aluminium, nanoparticle, isolated brain mitochondria, toxicity, oxidative stress.
\end{abstract}

\section{Introduction}

The use of nanomaterials in various applications is growing because of their physical, chemical and biological novel properties such as large specific surface area and high reaction activity (1). Aluminium oxide nanoparticles are one of the most plentifully produced nanoparticles (NPs) used in different fields such as catalysis, ceramics, polymer modification, heat transfer fluids, and waste water treatment. In addition, aluminium nanoparticles (AlNPs) have shown vast biological applications in biosensors, biofiltration, drug delivery and antigen delivery for immunization purposes (2). Therefore, there is a potential risk for discharging AlNPs into environment. Nowadays, neurotoxicity of aluminium

${ }^{1}$ Pharmaceutical Science Research Center, Hemoglobinopathy Institute, Mazandaran University of Medical Sciences, Sari, Iran, ${ }^{2}$ Department of Toxicology and Pharmacology, Faculty of Pharmacy, Mazandaran University of Medical Sciences, Sari, Iran, and ${ }^{3}$ Department of Toxicology and Pharmacology, Faculty of Pharmacy, Guilan University of Medical Sciences, Rasht, Iran

Address for correspondence: F. Shaki, PhD, Khazarabad road, Faculty of Pharmacy, Mazandaran University of Medical Sciences, Sari, Iran. postal code: 48471-93696.

Phone: +98.9112559051
(Al) has gained an increasing attention because of clinical and experimental evidence in humans and rodents (3). It has been shown that $\mathrm{Al}$ can accumulates in all regions of rat brain following chronic exposure, while reaching its maximum in the hippocampus, which is the site of memory and learning $(4,5)$. Also, neurological dysfunctions that were reported in dialysis patients were probably related to high $\mathrm{Al}$ concentrations in the dialysis fluid as well as to the use of phosphate-binding gels containing $\mathrm{Al}$ (6). In addition, several studies exhibited that Al-contaminated drinking water is a risk factor for Alzheimer's disease (7-9).

It has been shown that the ability of NPs to induce oxidative stress is one of the most important mechanisms involved in their toxic effects. In fact, NPs can induce reactive oxygen species (ROS) directly via exposure to acidic environment of lysosomes (10) or via interacting with oxidative organelles such as mitochondria (11).

Previous studies showed that Al exposure is associated with the impairment of mitochondrial functions both in vitro (12) and in vivo (13). Mitochondria are the major source of ROS and energy production in cells. Toxic materials can affect the electron transport chain (ETC) and lead to increased ROS production and oxidation of mitochondrial DNA, proteins and lipids. These events lead to the opening of mitochondrial permeability transition (MPT) pores and thus initiate the cell death signalling (14). 
The present study aims to provide a comprehensive analysis of the toxic effect of AlNPs on isolated rat brain mitochondria and compare it with that of aluminum ionic form.

\section{Material and methods}

\section{Animals}

Male Wistar rats (250-300 g), were provided from Laboratory Animals Research Center, Mazandaran University of Medical Sciences, Sari, Iran. Animals were housed in an air-conditioned room with controlled temperature of $22 \pm 2{ }^{\circ} \mathrm{C}$ and maintained on a $12 / 12-\mathrm{h}$ light/dark cycle with free access to food and water. All experimental procedures were conducted according to the ethical standard and protocols approved by the Committee of Animal Experimentation of Mazandaran University of Medical Sciences, Sari, Iran.

All efforts were made to minimize the number of animals used and their suffering.

\section{Mitochondrial preparation}

Mitochondria were prepared from the whole brain of Wistar rats using the differential centrifugation technique (15). Protein concentrations were determined by the Coomassie blue protein-binding method using bovine serum albumin as standard (16). The isolation of mitochondria was confirmed by measuring succinate dehydrogenase (17). Isolated brain mitochondria were prepared fresh for each experiment and used within $4 \mathrm{~h}$ after isolation and all steps were strictly operated on ice. Mitochondrial protein concentration of 1 $\mathrm{mg} / \mathrm{ml}$ was used for normalization of all experiments. Isolated brain mitochondria were incubated with different concentrations of AlNPs and aluminium ions (AlIs) $(50,100$ and $200 \mu \mathrm{M})$ for $1 \mathrm{~h}$ at $37^{\circ} \mathrm{C}$.

\section{Measurement of reactive oxygen species}

The ROS level was measured using dichlorofluorescin-diacetate (DCFH-DA) through a Shimadzu RF5000U fluorescence spectrophotometer at $485 \mathrm{~nm}$ excitation and $520 \mathrm{~nm}$ emission wavelength (18) .

For finding the source of ROS production in the electron transfer chain in mitochondria, malate/pyruvate (substrate complex I) and succinate (substrate complex III) were used in incubation medium as two different substrates, while in some samples, 2 $\mathrm{mM}$ rotenone (inhibitor complex I), $2 \mathrm{mM}$ antimycin $\mathrm{A}$ (inhibitor complex III) were added (15).

\section{Measurement of Lipid peroxidation (LPO)}

The content of malondialdehyde (MDA), as a marker of lipid peroxidation, was determined using the dithiobarbituric acid (TBA) method (19).

\section{Measurement of glutathione content}

The glutathione (GSH) content was determined by 5,5'-dithiobis-2-nitrobenzoic acid (DTNB) as an indicator. (20).

\section{Measurement of protein carbonyl}

The determination of protein carbonyl was performed by using guanidine hydrochloride reagent (21).

\section{Assessment of mitochondrial toxicity}

Mitochondrial toxicity was assessed by measuring the reduction of tetrazolium salt (MTT). (22).

Determination of the mitochondrial membrane potential (MMP)

Mitochondrial uptake of the cationic fluorescent dye, rhodamine 123, has been used to estimate the mitochondrial membrane potential. The fluorescence was monitored using Schimadzou RF-5000U fluorescence spectrophotometer at the excitation and emission wavelength of $490 \mathrm{~nm}$ and $535 \mathrm{~nm}$, respectively (23).

\section{Determination of mitochondrial swelling}

The mitochondrial swelling in the isolated brain mitochondria was estimated through changes in light scattering monitored spectrophotometrically at $540 \mathrm{~nm}\left(30^{\circ} \mathrm{C}\right)(24)$.

\section{Cytochrome c release assay}

The concentration of cytochrome c was determined by using the Quantikine rat/mouse cytochrome c immunoassay kit provided by R \& D Systems, Inc. (Minneapolis, Minn.).

\section{Statistical analysis}

All results are expressed as mean $\pm \mathrm{SEM}$. The distribution of our data follows a normal pattern. The significance of difference between two groups was evaluated using unpaired and paired Student's t-test. For multiple comparisons, one-way analysis of variance (ANOVA) was used. When ANOVA showed a significant difference, Tukey's post-hoc test was applied. The statistical significance was regarded when $\mathrm{p}<0.05$.

\section{Results}

\section{Effects of AlNPs and AlIs on ROS production}

As presented in Figure 1, the addition of both AlNP and AlI to isolated brain mitochondria resulted in an increase in ROS generation in a concentration-dependent manner. Next, the effect of AlNPs on mitochondrial electron transfer chain was evaluated by measuring ROS generation with complex substrates I and III. As shown in Table 1, ROS generation was increased after adding AlNPs in the presence of malate/pyruvate (complex substrate I) and succinate (complex substrate III). As a result, AlNP-induced ROS production increased more in succinate-supported mitochondria than that observed after adding malate/pyruvate. The ROS production was also measured alternatively with complex

Tab. 1. Effect of complex substrate I and III and inhibitors on AINP -induced ROS production.

\begin{tabular}{lcc}
\hline Treatment & + malate/pyruvate & + succinate \\
\hline Control & $58 \pm 6.2$ & $54 \pm 7.1$ \\
AlNP & $108 \pm 11.5^{*}$ & $120 \pm 8.4^{*}$ \\
AlNP 10mM+Antimycin A & $146 \pm 9.7^{\#}$ & $165 \pm 11.6^{\#}$ \\
AlNP 10mM+Rotenone & $125.3 \pm 8^{\#}$ & $128.3 \pm 7.4$ \\
\hline
\end{tabular}

Values are expressed as mean \pm SD for three rats in each group. * Significantly different when compared to the control $(\mathrm{p}<0.05)$, \# significantly different when compared to AlNP-treated mitochondria $(\mathrm{p}<0.05)$. 


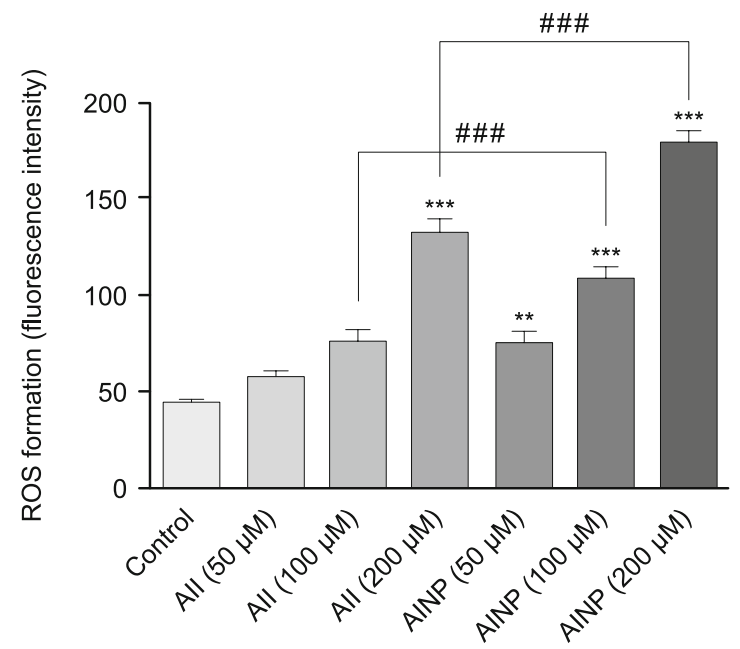

Fig. 1. Effects of aluminium ions (AIIs) and aluminium nanoparticles (AINPs) on reactive oxygen species (ROS) production in isolated brain mitochondria. Data were expressed as mean \pm standard error. The reactive oxygen species production was evaluated by dichlorofluorescein as an indicator as described in Material and Methods. ** Significantly different from control group $(p<0.01), * * *$ significantly different from control group $(p<0.001),{ }^{\# \#}$ significantly different from AlI group.

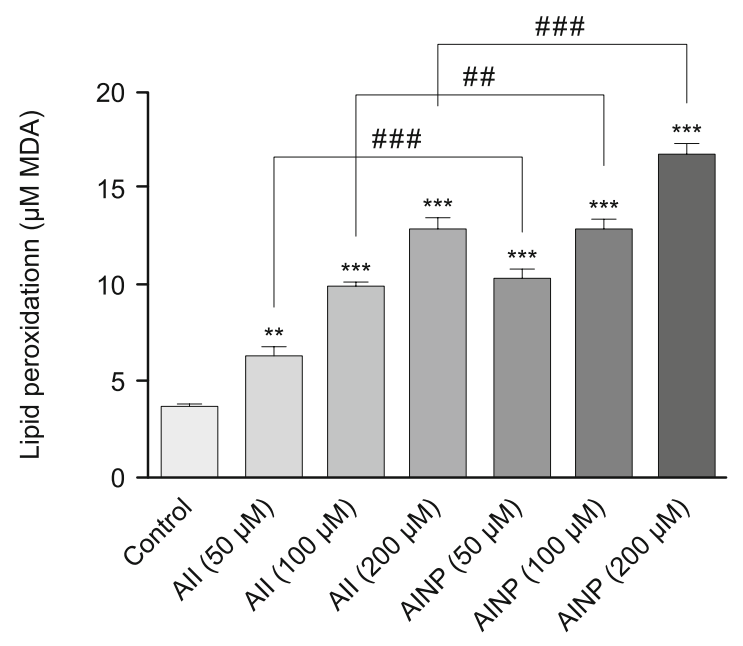

Fig. 2. Effects of aluminium ions (AIIs) and aluminium nanoparticles (AINPs) on lipid peroxidation in isolated brain mitochondria. Data were expressed as mean \pm standard error. Lipid peroxidation was evaluated by malondialdehyde (MDA) level as an indicator as described in Material and methods. ** Significantly different from control group $(p<0.01), * * *$ significantly different from control group $(p<0.001)$, \#\# significantly different from AlI group $(p<0.01)$, ${ }^{\# \#}$ significantly different from AlI group $(p<0.001)$.

inhibitors I and III being either present or absent.. The addition of antimycin A (inhibitor complex III) elevated the AINP-induced ROS production and in contrast to the latter complex, the addition of rotenone showed no significant effect on AlNP-induced ROS production in succinate-supported mitochondria. However, in the presence of AINPs, rotenone induced a high rate of ROS production in malate/pyruvate-supported mitochondria.

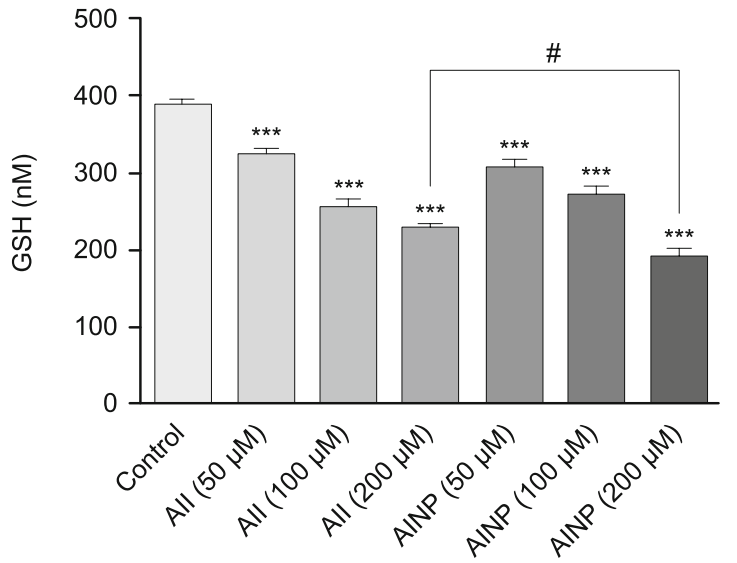

Fig. 3. Effects of aluminium ions (AIIs) and aluminium nanoparticles (AINPs) on glutathione (GSH) level in isolated brain mitochondria. Data were expressed as mean \pm standard error. GSH was evaluated by DTNB level as an indicator as described in Material and Methods. $* *$ Significantly different from control group $(p<0.001)$, significantly different from AlI group $(p<0.05)$.

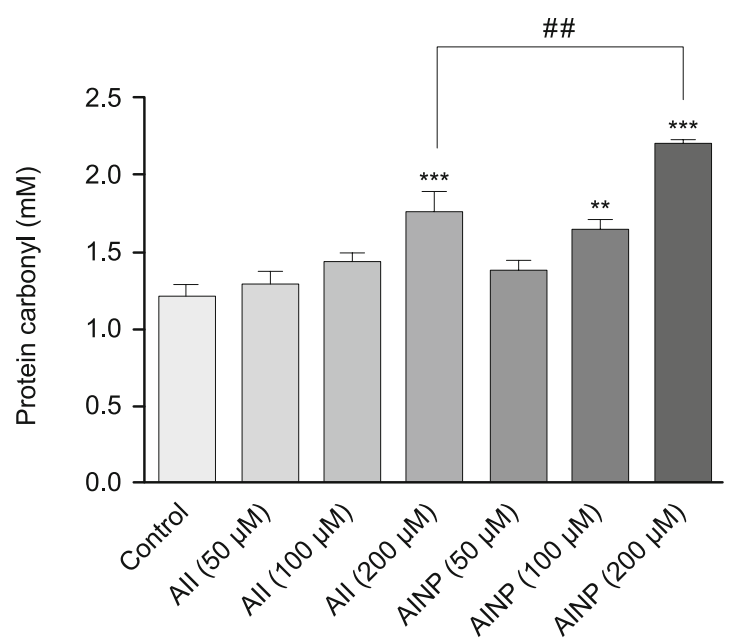

Fig. 4. Effects of aluminium ions (AIIs) and aluminium nanoparticles (AINPs) on protein carbonyl concentration in isolated brain mitochondria. Data were expressed as mean \pm standard error. Protein carbonyl was determined by reading the absorbance at $365 \mathrm{~nm}$ wave length as described in Material and Methods. **significantly different from control group $(\mathbf{p}<\mathbf{0 . 0 1}),{ }^{* * *}$ significantly different from control group $(\mathrm{p}<0.001),{ }^{\#}$ significantly different from AII group $(\mathrm{p}<0.01)$

\section{Effects of AlNPs and AlIs on lipid peroxidation}

As shown in Figure 2, the exposure of brain mitochondria to AlIs and AlPs increased the MDA values to a significantly higher level than was that measured in the control group $(\mathrm{p}<0.05)$. Also, the administration of AlNPs to brain mitochondria increased the MDA values to a significantly higher level than was that determined in AlI group $(\mathrm{p}<0.05)$. 
Effects of AlNPs and AlIs on glutathione levels

The GSH concentration was found to be decreased in consequence of ROS formation in groups treated with AlIs and AlNPs. However, the depletion of GSH was significant after the administration of AlIs and AlNPs at all concentrations. Also, as shown in Figure 3, AlNPs (at $200 \mu \mathrm{M}$ ) significantly affected the oxide GSH level in isolated brain mitochondria as compared to that in AlI group $(\mathrm{p}<0.05)$.

\section{Effects of AlNPs and AlIs on protein carbonyl production}

In this study, a high concentration of AlIs $(200 \mu \mathrm{M})$ significantly raised the protein carbonyl concentration in isolated brain mitochondria $(p<0.05)$. The raising effect on the latter concentration can be induced also by lower concentrations of AlNPs (100 and $200 \mu \mathrm{M}$ ), When comparing the effects of the same concentrations of AlNPs and AlIs, namely that of $200 \mu \mathrm{M}$, the promotion of protein carbonyl induced by the former agent was significantly higher $(\mathrm{p}<0.05)$ (Fig. 4).

\section{Effects of AlNPs and AlIs on mitochondrial function}

As compared to the control group, the administration of AlNPs and AlIs at concentrations $100 \mu \mathrm{M}$ and $200 \mu \mathrm{M}$ significantly decreased the function of brain mitochondria in a concentrationdependent manner $(p<0.05)$. As to the comparison of the effects of AlNPs and AlIs, we found out that at concentration of $200 \mu \mathrm{M}$, the former agent was significantly more effective in attenuating the mitochondrial function (Fig. 5).

Effects of AlNPs and AlIs on mitochondrial membrane potential

As shown in Figure 7, AlNPs significantly increased the MMP in a concentration-related manner $(p<0.05)$. Additionally, there is a significant difference between AlNP and AlI at 100 and 200 $\mu \mathrm{M}$ concentrations (Fig 6).

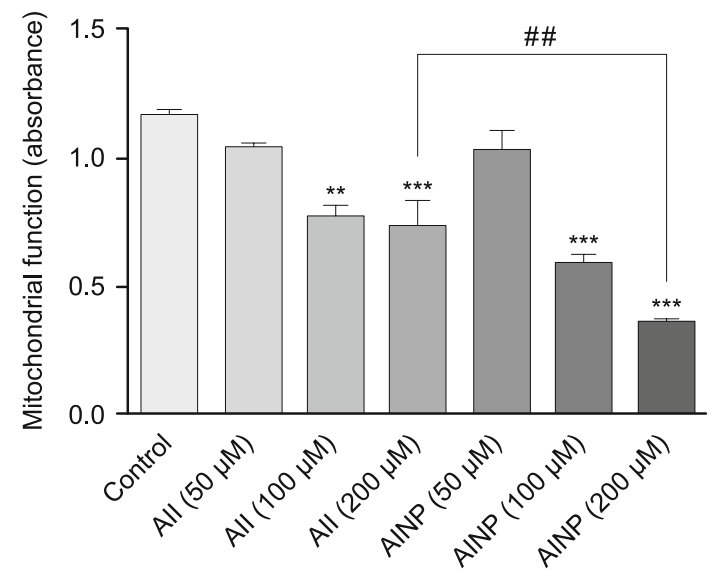

Fig. 5. Effects of aluminium ions (AIIs) and aluminium nanoparticles (AINPs) on mitochondrial function. Data were expressed as mean \pm standard error. Mitochondrial function determined by measuring the reduction of MTT as described in Material and Methods. ** significantly different from control group $(\mathbf{p}<\mathbf{0 . 0 1})$, *** significantly different from control group ( $p<0.001)$, ${ }^{\#}$ significantly different from AlI group $(\mathbf{p}<0.01)$.

\section{Effects of AlNPs and AlIs on mitochondrial swelling}

The data in Figure 7 indicate that whereas the addition of AlNP at medium and high concentrations $(100$ and $200 \mu \mathrm{M})$ to mitochondrial suspensions led to a significant level of mitochondrial swelling in a concentration-dependent manner, the suppressing effect of AlNPs on mitochondrial absorbance at $540 \mathrm{~nm}$ was not as profound. The latter effect occurred to be significant only at the high concentration of AlNPs.

\section{Effects of AINP and AlI on cytochrome c release}

As to the quantity of cytochrome c, there were statistically significant differences between the control mitochondria and

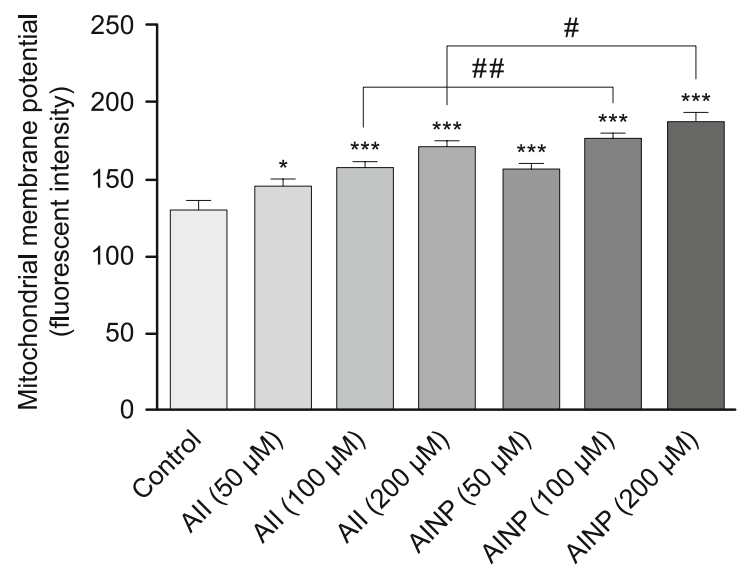

Fig. 6. Effects of aluminium ions (AIIs) and aluminium nanoparticles (AINPs) on mitochondrial membrane potential (MMP). Data were expressed as mean \pm standard error. MMP determined by Rhodamine 123 as an indicator as described in Material and methods. * significantly different from control group $(\mathbf{p}<0.05), * * *$ significantly different from control group $(p<0.001),{ }^{,}$significantly different from AII group $(p<0.05)$, significantly different from AlI group $(p<0.001)$.

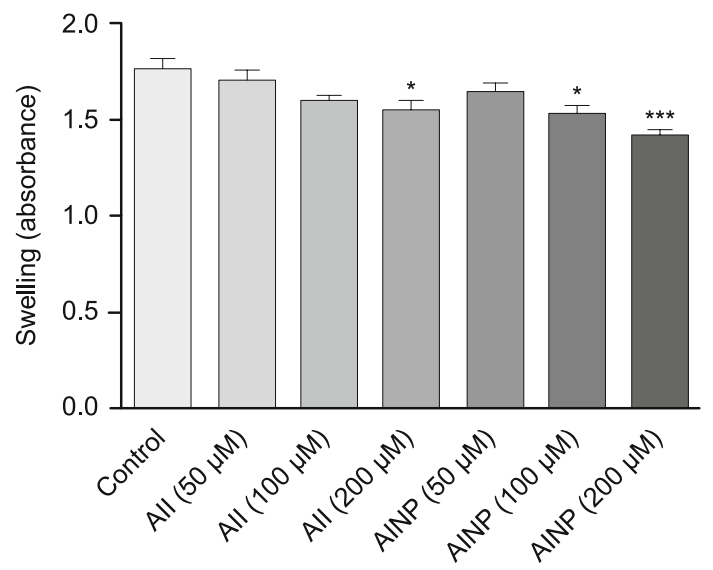

Fig. 7. Effects of aluminium ions (AIIs) and aluminium nanoparticles (AINPs) on mitochondrial swelling. Data were expressed as mean \pm standard error. Mitochondrial swelling was determined by reading the absorbance at $540 \mathrm{~nm}$ wave length as described in Material and Methods. * significantly different from control group $(\mathbf{p}<0.05)$, *** significantly different from control group $(p<0.001)$. 


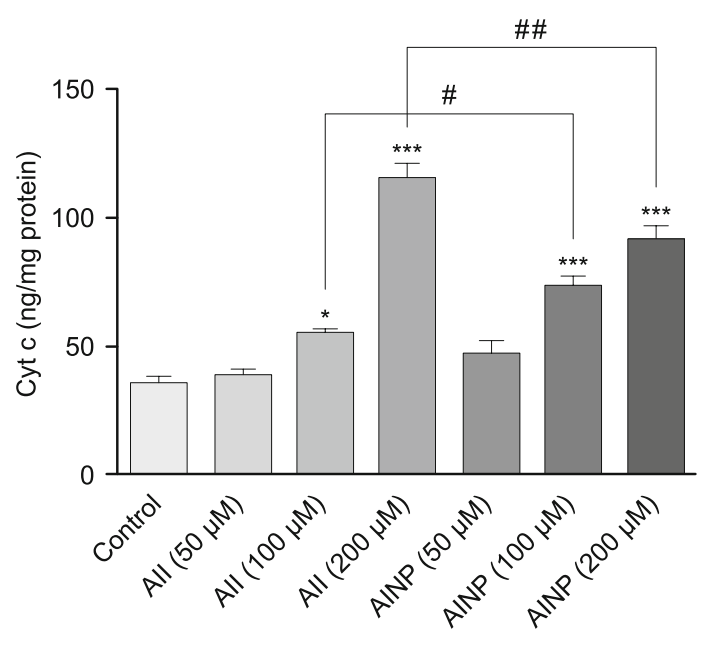

Fig. 8. Effect of aluminium ions (AIIs) and aluminium nanoparticles (AINPs) on cytochrome $\mathrm{c}$ release. Cytochrome $\mathrm{c}$ release was measured using cytochrome $\mathrm{c}$ assay kit as described in Material and Methods. Values represented as mean \pm standard error. * significantly different from control group $(p<0.05), * * *$ significantly different from control group $(p<0.001)$, " significantly different from AlI group $(p<0.05)$, \#\# significantly different from AII group $(p<0.01)$.

AlNP- and AlI-treated mitochondria, namely at concentrations of 100 and $200 \mu \mathrm{M}(\mathrm{p}<0.05)$. We also found out that following the administration of AlIs and AlNPs, the average concentration of expelled cytochrome $\mathrm{c}$ from mitochondrial fraction was amplified in a concentration-dependent manner. In addition, the administration of AlNPs to isolated mitochondria showed a significant statistical difference between AlNP and AlI groups, namely at concentrations of 100 and $200 \mu \mathrm{M}$ (Fig. 8).

\section{Discussion}

This study provides a detailed evaluation of mitochondrial toxicity of AlNPs at different concentrations. It determines their toxic effects on isolated brain mitochondria by measuring the mitochondrial oxidative damage endpoints and cell death pathway. At the same time, it compares the effects of AlNPs with those of aluminium's ionic form. Nowadays, there are several reports expressing health and environmental concerns related to the use of nanoparticles (25). AlNPs are among the most intensively produced nanomaterials. Their use is gaining momentum in a wide range of industries and biological applications (26). Previous studies showed increased incidence of neuro-degeneration diseases following exposure to $\mathrm{Al}$ (27). Also, oxidative stress and mitochondrial dysfunction is the most important mechanism suggested to take place in Al toxicity (27-29). It has been well shown that the mechanism of oxidative stress is commonly responsible for toxic effects of nanoparticles $(30,31)$. Mitochondria are known to be the main source of ROS generation in cells and various studies showed that nanoparticles of various sizes and chemical compositions could be preferentially localized in mitochondria while promoting ROS production $(32,33)$. For example, Wang et al reported the deposition of $\mathrm{CuO}$ nanoparticles in the mitochondria of human lung epithelial cells, which subsequently led to enhanced ROS production (34).

As shown in results, the exposure to both AINPs and AlIs induced ROS generation in a concentration-dependent manner. Previous studies revealed that complexes I and III are the main sources of ROS production in the mitochondrial respiratory chain (35). As evident in results, the addition of AlNPs significantly increased ROS production in both succinate and malate/pyruvate-supported brain mitochondria. It is worth mentioning that ROS production in succinate-supported mitochondria was more pronounced than in the malate/pyruvate-supported group. Also, the addition of antimycin A (an inhibitor of electron transport at the ubiquinone-cytochrome $b$ region) induced an elevation of ROS production while rotenone did not inhibit the succinate-supported ROS production. Evidence suggests that the ubiquinone-cytochrome $\mathrm{b}$ region in complex III can be considered as the main site of ALNP-induced ROS production in isolated brain mitochondria.

On the other hand, the brain has a high mitochondrial content and low level of antioxidant enzymes. Therefore, any agent that disrupts the brain mitochondrial function can lead to excessive ROS production. Therefore, at first, we evaluated the mitochondrial function after the exposure to both forms of aluminium (nanoparticles and ions) by means of measuring MTT. As shown in results, AlNPs induced mitochondrial toxicity in all concentrations and it was more pronounced than that induced by AlIs, thus demonstrating high mitochondrial toxicity of AlNPs.

In addition, the mitochondrial electron transfer chain is involved in energy as well as ROS production in cells. Interestingly, the release of cytochrome $\mathrm{c}$ from mitochondria can be the starting point of programmed cell death (apoptosis) signalling (36). It is well known that xenobiotics can promote ROS production via disruption of mitochondrial electron transfer chain. In addition, the brain is highly dependent on oxidative phosphorylation as its energy source compared to other cells in the body. Therefore, any agent that impairs the mitochondrial electron transfer can disturb ATP production as well as the normal pathway of electrons in mitochondria, which leads to increased ROS production and finally incurs oxidative damage to neurons (37). Also, several studies reported the important role of mitochondrial dysfunction in many neurodegenerative diseases $(38,39)$. Results indicated that AINPs have a more toxic effect than AlIs on isolated brain mitochondria in inducing oxidative stress parameters such as ROS, LPO, and protein carbonyl formation, including GSH oxidation.

It was previously reported that AINP can promote oxidative stress in various biological systems. For example, M'rad et al showed that $\mathrm{Al}_{2} \mathrm{O}_{3}$ nanoparticles could induce oxidative damage in the hipocampus by increasing he levels of MDA as well as by decreasing the levels of antioxidant defence enzymes such as superoxide dismutase and gluthation peroxidase (40).

In fact, it is believed that compared to the bulk material, NPs could induce more ROS production as a consequence of their large surface area (41). Also, several studies evaluated the ability of metal oxide NPs to induce oxidative stress in various experimental models, in which the amounts of ROS observed in metal oxide NPs' suspensions were higher than in ionic formulations (42). 
In this study, the exposure of isolated brain mitochondria to both $\mathrm{Al}$ forms significantly increased the lipid peroxidation. It has been shown that oxidation of lipid membrane results in disruption of mitochondrial membrane and consequently to the collapse of MMP and cytochrome c release (43). On the other hand, GSH oxidation was observed after exposure to different concentrations of AINP in isolated brain mitochondria. Not only are the reduced levels of GSH in mitochondria considered as the main antioxidant, they are also crucial for the maintenance of the reduced form of thiol groups in mitochondrial membrane proteins (44) Oxidation of these thiol groups facilitates conformational changes occurring in the pore complex that induces the mitochondrial permeability transition (MPT). The opening of MPT pores permits unlimited movement of water and solutes and mitochondria undergo structural changes referred to as mitochondrial swelling (15). Accordingly, we investigated the effect of AINPs on MMP and mitochondrial swelling. As shown in the results, AlNPs decreased MMP and induced mitochondrial swelling in a concentration-dependent manner. Interestingly, AlNPs showed significantly more toxic effects in inducing MMP and mitochondrial swelling than AlIs. The induction of MPT can be associated with the release of apoptogenic factor such as cytochrome $\mathrm{c}$ into cytosol which then initiates both necrosis and apoptosis mechanisms (45). Accordingly, in comparison with AlIs, the AlNPs induced a significantly higher release of cytochrome $\mathrm{c}$ from isolated brain mitochondria. The latter fact emphasises the confirmed mitochondrial toxicity of these nanoparticles.

\section{Conclusion}

This study showed that the mechanism of AlNPs' toxicity in isolated brain mitochondria takes place via the inhibition of electron transfer chain. Also, AlNPs increased the inner membrane permeability and mitochondrial membrane potential dissipation, thus leading to a release of apoptogenic factors. In overall, most of the mitochondrial toxicity endpoints in the present study showed that the effect of AlNPs was more toxic than that of aluminium's ionic form, which can be explained by high reactivity of NPs due to their large surface area.

\section{References}

1. Park E-J, Lee G-H, Shim J-h, Cho M-H, Lee B-S, Kim Y-B et al. Comparison of the toxicity of aluminum oxide nanorods with different aspect ratio. Arch Toxicol 2015; 89 (10): 1771-1782.

2. Kumar V, Gill KD. Oxidative stress and mitochondrial dysfunction in aluminium neurotoxicity and its amelioration: a review. Neurotoxicology 2014; 41: 154-166.

3. Becaria A, Campbell A, Bondy S. Aluminum as a toxicant. Toxicol Industr Health 2002; 18 (7): 309-320.

4. Kaur A, Joshi K, Minz RW, Gill KD. Neurofilament phosphorylation and disruption: a possible mechanism of chronic aluminium toxicity in Wistar rats. Toxicology 2006; 219 (1-3): 1-10.

5. Sánchez-Iglesias S, Soto-Otero R, Iglesias-Gonzalez J, BarcielaAlonso MC, Bermejo-Barrera P, Méndez-Álvarez E. Analysis of brain regional distribution of aluminium in rats via oral and intraperitoneal administration. J Trace Element Med Biol 2007; 21: 31-34.

6. Jack R, Rabin PL, McKinney TD. Dialysis encephalopathy: a review. Internat J Psychiat Med 1984; 13 (4): 309-326.

7. Neri L, Hewitt D. Aluminium, Alzheimer's disease, and drinking water. Lancet 1991; 338 (8763): 390.

8. Gauthier E, Fortier I, Courchesne F, Pepin P, Mortimer J, Gauvreau D. Aluminum forms in drinking water and risk of Alzheimer's disease. Environ Res 2000; 84 (3): 234-246.

9. Flaten TP. Aluminium as a risk factor in Alzheimer's disease, with emphasis on drinking water. Brain Res Bull 2001; 55 (2): 187-196.

10. Nohl H, Gille L. Lysosomal ROS formation. Redox Report. 2005; 10 (4): 199-205.

11. Zhang DX, Gutterman DD. Mitochondrial reactive oxygen speciesmediated signaling in endothelial cells. Amer J Physiol Heart Circ Physiol 2007; 292 (5): H2023-H31.

12. Niu $P$, Niu Q, Zhang $Q$, Wang $L$, He $S$, Wu T, et al. Aluminum impairs rat neural cell mitochondria in vitro. Internat J Immunopathol Pharmacol 2005; 18 (4): 683-689.

13. Kumar V, Bal A, Gill KD. Impairment of mitochondrial energy metabolism in different regions of rat brain following chronic exposure to aluminium. Brain Res 2008; 1232: 94-103.

14. Halliwell B. Reactive oxygen species and the central nervous system. Free radicals in the brain: Springer; 1992, 21-40.

15. Shaki F, Hosseini M-J, Ghazi-Khansari M, Pourahmad J. Depleted uranium induces disruption of energy homeostasis and oxidative stress in isolated rat brain mitochondria. Metallomics.2013; 5 (6): 736-744.

16. Vahidirad M, Arab-Nozari M, Mohammadi H, Shaki F. Protective Effect of Edaravone against Nephrotoxicity and Neurotoxicity of Acute Exposure to Diazinon. J Mazandaran Univ Med Sci 2018; 28 (165): 175-182.

17. Shokrzadeh M, Alidoust F, Nourian Y, Vaezi N, Mohammadi E, Shaki F. Protective Effects of Resveratrol against Paraquat-Induced Mitochondrial Dysfunction in Brain and Lung Isolated Mitochondria. J Mazandaran Univ Med Sci 2014; 24 (115).

18. Vahidirad M, Arab-Nozari M, Mohammadi H, Zamani E, Shaki F. Protective effect of captopril against diazinon induced nephrotoxicity and neurotoxicity via inhibition of ROS-NO pathway. Drug Chem Toxicol 2018; 41 (3): 287-293.

19. Zhang F, Xu Z, Gao J, Xu B, Deng Y. In vitro effect of manganese chloride exposure on energy metabolism and oxidative damage of mitochondria isolated from rat brain. Environ Toxicol Pharmacol 2008; 26 (2): 232-236.

20. Sadegh C, Schreck RP. The spectroscopic determination of aqueous sulfite using Ellman's reagent. MURJ 2003; 8: 39-43.

21. Hensley K, Hall N, Subramaniam R, Cole P, Harris M, Aksenov M, et al. Brain regional correspondence between Alzheimer's disease histopathology and biomarkers of protein oxidation. J Neurochem 1995; 65 (5): 2146-2156.

22. Shokrzadeh M, Zamani E, Mehrzad M, Norian Y, Shaki F. Protective effects of propofol against Methamphetamine-induced neurotoxicity. Toxicol Internat 2015; 22 (1): 92.

23. Hosseini M-J, Shaki F, Ghazi-Khansari M, Pourahmad J. Toxicity of vanadium on isolated rat liver mitochondria: a new mechanistic approach. Metallomics 2013; 5 (2): 152-166. 


\section{6-522}

24. Hosseini M-J, Shaki FS, Ghazi-Khansari M, Pourahmad J. Toxicity of arsenic (III) on isolated liver mitochondria: a new mechanistic approach. Iran J Pharm Res 2013; 12: 121-138.

25. Abdel-Khalek AA, Kadry MA, Badran SR, Marie M-AS. Comparative toxicity of copper oxide bulk and nano particles in Nile tilapia; Oreochromis niloticus: biochemical and oxidative stress. J Basic Appl Zool 2015; 72: 43-57.

26. Yang S-T, Wang T, Dong E, Chen X-X, Xiang K, Liu J-H, et al. Bioavailability and preliminary toxicity evaluations of alumina nanoparticles in vivo after oral exposure. Toxicol Res 2012; 1 (1): 69-74.

27. Wu Z, Du Y, Xue H, Wu Y, Zhou B. Aluminum induces neurodegeneration and its toxicity arises from increased iron accumulation and reactive oxygen species (ROS) production. Neurobiol Aging 2012; 33 (1): 199.e1-e12.

28. Kawahara M, Kato-Negishi M. Link between aluminum and the pathogenesis of Alzheimer's disease: the integration of the aluminum and amyloid cascade hypotheses. Internat J Alzheimer Dis 2011; 2011.

29. Lukiw WJ, Pogue AI. Induction of specific micro RNA (miRNA) species by ROS-generating metal sulfates in primary human brain cells. J Inorganic Biochem 2007; 101 (9): 1265-1269.

30. Carlson C, Hussain SM, Schrand AM, K. Braydich-Stolle L, Hess KL, Jones RL et al. Unique cellular interaction of silver nanoparticles: size-dependent generation of reactive oxygen species. J Phys Chem B 2008; 112 (43): 13608-13619.

31. Suliman Y, Omar A, Ali D, Alarifi S, Harrath AH, Mansour L et al. Evaluation of cytotoxic, oxidative stress, proinflammatory and genotoxic effect of silver nanoparticles in human lung epithelial cells. Environ Toxicol 2015; 30 (2): 149-160.

32. AshaRani P, Low Kah Mun G, Hande MP, Valiyaveettil S. Cytotoxicity and genotoxicity of silver nanoparticles in human cells. ACS nano 2008; 3 (2): 279-290.

33. Vrček IV, Žuntar I, Petlevski R, Pavičić I, Dutour Sikirić M, Ćurlin M et al. Comparison of in vitro toxicity of silver ions and silver nanoparticles on human hepatoma cells. Environ Toxicol 2014.
34. Wang Z, Li N, Zhao J, White JC, Qu P, Xing B. CuO nanoparticle interaction with human epithelial cells: cellular uptake, location, export, and genotoxicity. Chem Res Toxicol 2012; 25 (7): 1512-1521.

35. Barja G. Minireview: the quantitative measurement of $\mathrm{H} 2 \mathrm{O} 2$ generation in isolated mitochondria. J Bioenerg Biomembrane 2002; 34 (3): $227-233$.

36. Shaki F, Pourahmad J. Mitochondrial toxicity of depleted uranium: Protection by beta-glucan. Iran J Pharm Res 2012; 12 (1): 131-140.

37. Emerit J, Edeas M, Bricaire F. Neurodegenerative diseases and oxidative stress. Biomed Pharmacother 2004; 58 39-46.

38. Rego AC, Oliveira CR. Mitochondrial dysfunction and reactive oxygen species in excitotoxicity and apoptosis: implications for the pathogenesis of neurodegenerative diseases. Neurochem Res 2003; 28 (10): $1563-1574$

39. Islam MT. Oxidative stress and mitochondrial dysfunction-linked neurodegenerative disorders. Neurol Res 2017; 39 (1): 73-82.

40. M'rad I, Jeljeli M, Rihane N, Hilber P, Sakly M, Amara S. Aluminium oxide nanoparticles compromise spatial learning and memory performance in rats. EXCLI J 2018; 17: 200.

41. Stoeger T, Reinhard C, Takenaka S, Schroeppel A, Karg E, Ritter B et al. Instillation of six different ultrafine carbon particles indicates a surface area threshold dose for acute lung inflammation in mice. Environ Health Perspect 2006: 328-333.

42. Chang Y-N, Zhang M, Xia L, Zhang J, Xing G. The toxic effects and mechanisms of $\mathrm{CuO}$ and $\mathrm{ZnO}$ nanoparticles. Materials 2012; 5 (12): 2850-2871.

43. Ott M, Gogvadze V, Orrenius S, Zhivotovsky B. Mitochondria, oxidative stress and cell death. Apoptosis 2007; 12 (5): 913-922.

44. Shaki F, Pourahmad J. Mitochondrial toxicity of depleted uranium: Protection by beta-glucan. Iran J Pharm Res 2013; 12 (1): 131.

45. Garrido C, Galluzzi L, Brunet M, Puig P, Didelot C, Kroemer G. Mechanisms of cytochrome c release from mitochondria. Cell Death Differentiation 2006; 13 (9): 1423-1433. 